EPJ Web of Conferences 60, 20040 (2013)

DOI: $10.1051 /$ epjconf $/ 20136020040$

(C) Owned by the authors, published by EDP Sciences, 2013

\title{
Search for direct production of charginos and neutralinos in events with three leptons and missing transverse momentum in $21 \mathrm{fb}^{-1}$ of $\mathrm{pp}$ collisions at $\sqrt{(s)}=8 \mathrm{TeV}$ with the ATLAS detector
}

\author{
Basil Schneider ${ }^{1, a}$ on behalf of the ATLAS Collaboration. \\ ${ }^{1}$ University of Bern
}

\begin{abstract}
A search for direct chargino and neutralino production processes is presented in a final state with exactly 3 leptons (electrons or muons). No excess over the Standard Model has been observed. The analysis presented is based on $20.7 \mathrm{fb}^{-1}$ of proton-proton collision data delivered by the LHC at $\sqrt{(s)}=8 \mathrm{TeV}$ and recorded by the ATLAS [2] detector in 2012. [1]
\end{abstract}

\section{Introduction}

Supersymmetry (SUSY) postulates the existence of SUSY particles with spin differing by one-half unit with respect to that of their Standard Model (SM) partner. SUSY events with multilepton final states can effectively be separated by ATLAS [2] from the overwhelming hadron-rich SM background typical of high-energy hadron-hadron collisions. Assuming colored SUSY particles are heavy, electroweakly produced gaugino production then becomes the dominant SUSY cross-section at the LHC. For the gaugino decays, a high lepton multiplicity and a missing transverse momentum from the escaping least supersymmetric particles is expected, while there is no hadronic activity, which makes this signature very clean.

Simplified SUSY models have the least particle content necessary to mimic a SUSY like event. No assumptions about relative couplings or interference terms are made, this provides very generic results which can be applied to any theories with additional SM partner particles leading to the same signature.

In this analysis, simplified models with associated production of the lightest chargino $\left(\tilde{\chi}_{1}^{ \pm}\right)$and the secondlightest neutralino $\left(\tilde{\chi}_{2}^{0}\right)$ are considered. Both are assumed to be wino-like with a small higgsino component and with a degenerate mass. The decay of those two SUSY particles can happen via intermediate gauge bosons (Fig. 1 (a)) or via intermediate sleptons (Fig. 1 (b)), whose masses are assumed to be midway between $\tilde{\chi}_{1}^{0}$ and $\tilde{\chi}_{2}^{0}$.

\section{Signal regions}

Most of the SM background is suppressed by selecting final states with three isolated leptons (electrons or muons) and rejecting events with b-jets. Further requirements are

\footnotetext{
a e-mail: basil.schneider@ cern.ch
}

applied to form 6 signal regions; variables used are the missing transverse energy, the invariant mass formed with the two same flavour opposite sign (SFOS) leptons closest to the $Z$ mass, the transverse mass formed with the missing transverse energy and the leftover lepton and the $p_{T}$ of the $3^{\text {rd }}$ lepton. A breakdown of the 6 signal regions used in this analysis is shown in Table 1. Different signal regions are targeting different parts of the simplfied model grids. The simplified model which decays via WZ (Fig. 1 (a)), along the diagonal line where $\tilde{\chi}_{2}^{0}-\tilde{\chi}_{1}^{0}=m_{Z}$, has a signature similar to $W Z$, the distinction between background and signal is difficult and therefore more signal regions are used to target this part of the model. It has been shown that these signal regions are optimal for selected reference signal points based on distributions of the discriminating variables in regions where all cuts of the signal region are applied except on one variable, in order to determine its optimal value.

\section{Background modeling}

SM background events with 3 prompt leptons (i.e. $W Z$, ZZ) are derived by Monte Carlo simulations; events with at least one non-prompt or non-genuinely isolated lepton are estimated with a data-driven matrix method. Before looking at data in the signal regions, the background modeling was tested in dedicated validation regions, which are chosen to be orthogonal to the SR's by inversing one cut. Excellent agreement between data and background modeling has been found. The definition of the validation regions can be seen in Table 2. Some examplary distributions are shown in Fig. 2.

\section{Results}

A breakdown of the expected and observed events in all 6 signal regions can be seen in Table 3. No significant ex- 


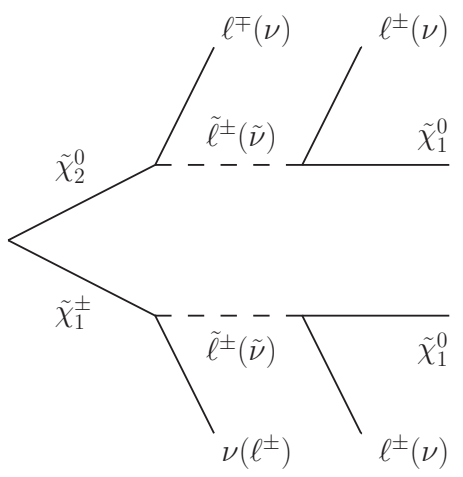

(a) Decay via intermediate sleptons

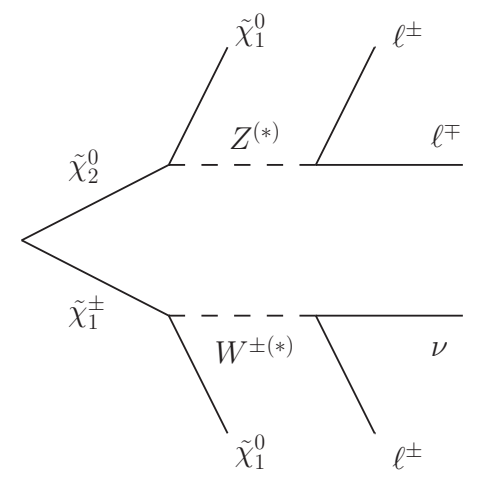

(b) Decay via intermediat gauge bosons

Figure 1. Diagrams for the two $\tilde{\chi}_{1}^{ \pm}, \tilde{\chi}_{2}^{0}$ simplified models studied in this note.

\begin{tabular}{lcccccc}
\hline Selection & SRnoZa & SRnoZb & SRnoZc & SRZa & SRZb & SRZc \\
\hline$m_{\mathrm{SFOS}}[\mathrm{GeV}]$ & $<60$ & $60-81.2$ & $<81.2$ or $>101.2$ & $81.2-101.2$ & $81.2-101.2$ & $81.2-101.2$ \\
$\mathbb{E}_{T}[\mathrm{GeV}]$ & $>50$ & $>75$ & $>75$ & $75-120$ & $75-120$ & $>120$ \\
$m_{\mathrm{T}}[\mathrm{GeV}]$ & - & - & $>110$ & $<110$ & $>110$ & $>110$ \\
$p_{T} 3^{\text {rd }} \ell[\mathrm{GeV}]$ & $>10$ & $>10$ & $>30$ & $>10$ & $>10$ & $>10$ \\
SR veto & SRnoZc & SRnoZc & - & - & - & - \\
\hline
\end{tabular}

Table 1. The selection requirements for the signal regions. All regions are mutually exclusive and require exactly three signal leptons and a same-flavour opposite-sign (SFOS) lepton pair. Events with a $b$-jet or a SFOS lepton pair with mass less than $12 \mathrm{GeV}$ are rejected. The mass of the SFOS lepton pair closest to the $Z$-boson mass is denoted by $m_{\mathrm{SFOS}}$. The $m_{\mathrm{T}}$ is calculated from the $\mathbb{E}_{T}$ and the lepton not forming the SFOS lepton pair closest to the $Z$-boson mass.

\begin{tabular}{lcccc}
\hline Selection & VRnoZa & VRnoZb & VRZa & VRZb \\
\hline$m_{\mathrm{SFOS}}[\mathrm{GeV}]$ & $<81.2$ or $>101.2$ & $<81.2$ or $>101.2$ & $81.2-101.2$ & $81.2-101.2$ \\
$b$-jet & veto & request & veto & request \\
$\mathbb{E}_{T}[\mathrm{GeV}]$ & $35-50$ & $>50$ & $30-50$ & $>50$ \\
Dominant process & $W Z^{*}, Z^{*} Z^{*}, Z^{*}+$ jets & $t \bar{t}$ & $W Z, Z+$ jets & $W Z$ \\
\hline
\end{tabular}

Table 2. The selection requirements of the validation regions. All regions require exactly three signal leptons and a same-flavour opposite-sign (SFOS) lepton pair. Events that contain a SFOS lepton pair with a mass less than $12 \mathrm{GeV}$ are rejected. The mass of the SFOS lepton pair closest to the $Z$-boson mass is denoted by $m_{\mathrm{SFOS}}$.

cess over the SM is observed. Therefore limits are set in the two simplified models. For large mass differences with the $\tilde{\chi}_{1}^{0}$, in the models with intermediate slepton decays, degenerate $\tilde{\chi}_{1}^{ \pm}$and $\tilde{\chi}_{2}^{0}$ masses up to $600 \mathrm{GeV}$ are excluded (see Fig. 3 (a)), while in the simplified models with intermediate gauge bosons, degenerate $\tilde{\chi}_{1}^{ \pm}$and $\tilde{\chi}_{2}^{0}$ masses up to $315 \mathrm{GeV}$ are excluded (see Fig. 3 (b)).

Observed and expected exclusion limits at the $95 \%$ confidence level for chargino and neutralino production are set by combining all 6 signal regions in a profile likelihood fit, where the systematic uncertainties are modeled as nuisance parameters.

Previous limits have been extended significantly.

\section{References}

[1] ATLAS Collaboration, ATLAS-CONF-2013-035 (https://cds.cern.ch/record/1532426/).

[2] ATLAS Collaboration, 2008 JINST 3 S08003. 


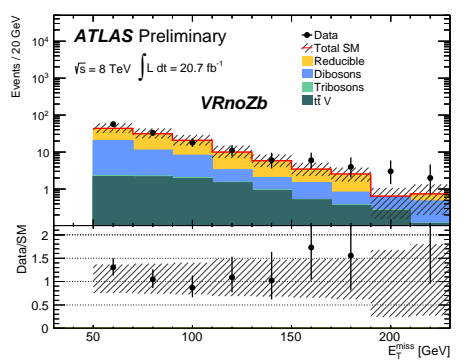

(a) Missing transverse energy in VRnoZb

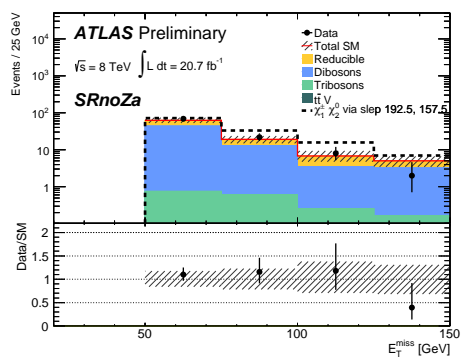

(c) Missing transverse energy in SRnoZa

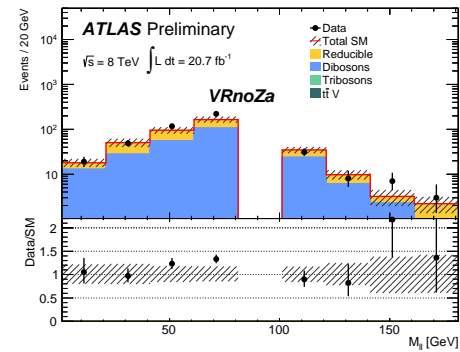

(b) $m_{S F O S}$ in VRnoZa

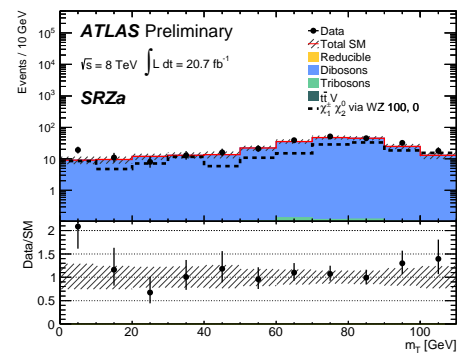

(d) Transverse mass in SRZa

Figure 2. Some distributions of different variables in validation regions $(\mathrm{a}, \mathrm{b})$ and signal regions $(\mathrm{c}, \mathrm{d})$. Excellent agreement between background modeling and data is observed, no excess is seen.

\begin{tabular}{lcccccc}
\hline Selection & SRnoZa & SRnoZb & SRnoZc & SRZa & SRZb & SRZc \\
\hline Tri-boson & $1.7 \pm 1.7$ & $0.6 \pm 0.6$ & $0.8 \pm 0.8$ & $0.5 \pm 0.5$ & $0.4 \pm 0.4$ & $0.29 \pm 0.29$ \\
$Z Z$ & $14 \pm 8$ & $1.8 \pm 1.0$ & $0.25 \pm 0.17$ & $8.9 \pm 1.8$ & $1.0 \pm 0.4$ & $0.39 \pm 0.28$ \\
$t \bar{t} V$ & $0.23 \pm 0.23$ & $0.21 \pm 0.19$ & $0.21_{-0.21}^{+0.30}$ & $0.4 \pm 0.4$ & $0.22 \pm 0.21$ & $0.10 \pm 0.10$ \\
$W Z$ & $50 \pm 9$ & $20 \pm 4$ & $2.1 \pm 1.6$ & $235 \pm 35$ & $19 \pm 5$ & $5.0 \pm 1.4$ \\
\hline$\Sigma$ SM irreducible & $65 \pm 12$ & $22 \pm 4$ & $3.4 \pm 1.8$ & $245 \pm 35$ & $20 \pm 5$ & $5.8 \pm 1.4$ \\
\hline SM reducible & $31 \pm 14$ & $7 \pm 5$ & $1.0 \pm 0.4$ & $4_{-4}^{+5}$ & $1.7 \pm 0.7$ & $0.5 \pm 0.4$ \\
\hline$\Sigma$ SM & $\mathbf{9 6} \pm \mathbf{1 9}$ & $\mathbf{2 9} \pm \mathbf{6}$ & $\mathbf{4 . 4} \pm \mathbf{1 . 8}$ & $\mathbf{2 4 9} \pm \mathbf{3 5}$ & $\mathbf{2 2} \pm \mathbf{5}$ & $\mathbf{6 . 3} \pm \mathbf{1 . 5}$ \\
\hline Data & $\mathbf{1 0 1}$ & $\mathbf{3 2}$ & $\mathbf{5}$ & $\mathbf{2 7 3}$ & $\mathbf{2 3}$ & $\mathbf{6}$ \\
\hline & & & & & &
\end{tabular}

Table 3. Expected numbers of events from SM backgrounds and observed numbers of events in data in the signal regions, for $20.7 \mathrm{fb}^{-1}$. Both statistical and systematic uncertainties are included.

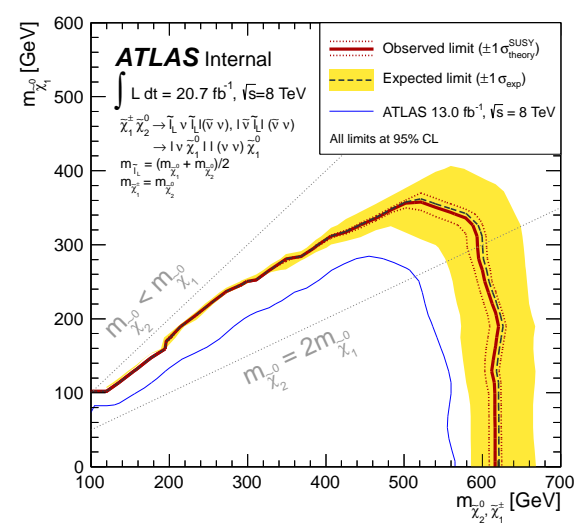

(a) Decay via sleptons

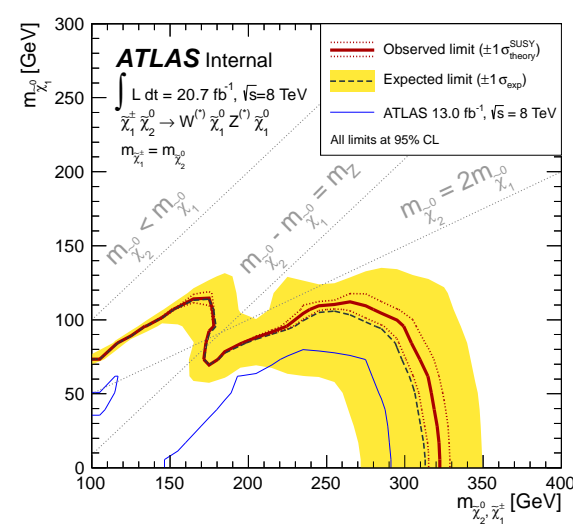

(b) Decay via gauge bosons

Figure 3. Observed and expected 95\% CL limit contours for chargino and neutralino production in the simplified model scenario with (a) decay via sleptons and (b) decay via gauge bosons. 